\title{
Radios comunitarias en Jujuy (Argentina): mapeo y condiciones de funcionamiento
}

\section{Community radios in Jujuy (Argentina): mapping and management}

structures

- Claudio Avilés Rodilla, Sonia Zegada

Universidad de Jujuy

\author{
Fecha de recepción: 25 de octubre de 2018 \\ Fecha de aprobación: 15 de diciembre de 2018
}

DOI: http://dx.doi.org/10.15304/ricd.2.9.5552

\section{NOTAS BIOGRÁFICAS}

Claudio Avilés Rodilla es doctor en Comunicación por la UNLP, docente en la UNJu y editor responsable de Viator, revista científica de comunicación desde los bordes y editor asistente en la revista Cuadernos.

\section{Contacto: claudiogaviles@gmail.com}

Sonia Zegada es licenciada en Comunicación Social por la UNJu y profesora en esta misma universidad. Coordina el proyecto de extensión "Radio del Viento, emisora experimental de producción radiofónica en internet".

Contacto: soniazegada@gmail.com

\section{Resumen}

En este artículo se presentan los resultados de una investigación en la que se aborda la situación del sector radiofónico comunitario en la provincia de Jujuy (Argentina) para caracterizar, entre otras cosas, los avances y los obstáculos que atravesaron los actores participantes locales. El objetivo general de la investigación fue relevar el universo de emisoras comunitarias que operan en la provincia Jujuy y analizar sus condiciones de funcionamiento, durante el periodo 2016 - 2017. Por otra parte, se sistematizaron y caracterizaron las estructuras de propiedad y estrategias de financiamiento de las radios comunitarias relevadas. Asimismo, se presentan los resultados y conclusiones que caracterizan y analizan las estructuras de gestión, organización y actores participantes en las distintas emisoras; y una clasificación panorámica de la situación de las emisoras comunitarias jujeñas en el desarrollo de programación y perfil de contenidos.

\section{Abstract}

This paper presents the results of an investigation in which the situation of the community radio sector in the province of Jujuy (Argentina) is analyzed to characterize, among other things, the advances and obstacles that the participating local actors went through. The general objective of the research was to survey the universe of community radio stations operating in the province of Jujuy and to check their operating conditions, during the period 2016 - 2017. On the other hand, 
property structures and financing strategies were systematized and characterized of the community radio stations surveyed. Likewise, the results and conclusions that characterize and analyze the management structures, organization and actors participating in the different stations are presented; and a panoramic classification of the situation of the community radio stations of Jujuy in the development of programming and content profile.

\section{Palabras clave}

Radios comunitarios, mapeo, propiedad, financiamiento, gestión.

\section{Keywords}

Community radios, mapping, proverty, financing, management.

\section{Sumario}

1. Introducción

2. Mapeo de emisoras comunitarias existentes en Jujuy (2016-2017)

3. Propiedad y estrategias de financiamiento de las emisores comunitarias

4. Formas de organización, gestión y actores participantes

5. Propuestas comunicativas y perfil de las emisoras

6. Conclusiones

\section{Contents}

1. Introduction

2. Mapping of community radio stations in Jujuy (2016-2017)

3. Ownership and financing strategies of community radio stations

4. Forms of organization, management and participating actors

5. Communication proposals and profile of the stations

6. Conclusions 


\section{INTRODUCCIÓN}

La radio y la televisión en Argentina se enmarcan en un contexto histórico atravesado por complejos vaivenes políticos y económicos, donde los poderes gubernamentales y empresariales jugaron papeles determinantes para configurar el escenario mediático actual.

Dicho escenario, hereditario de las políticas neoliberales de la década del noventa, presenta una estructura mayoritariamente privada e hipercomercial, con una escasa presencia de medios comunitarios, una menor existencia de medios estatales y una concentración de poder comunicacional con epicentro en los grandes centros urbanos.

El sector de radios comunitarias en Argentina surgió con la recuperación democrática y tuvo su principal expansión entre los años 1983 y 1986, al ritmo de la expansión de la banda de modulación de frecuencia (FM). Mientras que en la década neoliberal (1989-2001) puede identificarse con una depresión en la conformación de colectivos comunicacionales, asociado fundamentalmente a la continuidad y profundización de un marco regulatorio restrictivo para el sector (Iglesias, 2015).

Por ello, un hito fundamental en la historia fue la discusión y posterior sanción en 2009 de la Ley $n^{\circ} 26522$ de Servicios de Comunicación Audiovisual debido a que, por primera vez, un gobierno argentino democrático asumió una política pública que posicionó al Estado como garante de la comunicación como derecho. Específicamente, para el sector de emisoras comunitarias la ley suponía ${ }^{1}$ una reversión al histórico aplazamiento que sufrió en términos de reconocimiento y regulación, ya que, de manera inédita a nivel mundial, reservó el 33 por ciento de todo el espacio radioeléctrico a organizaciones sin fines de lucro y reconoció de manera explícita a dicho sector en el apartado "definiciones". Asimismo, la ley previó, en su artículo 97, herramientas de financiamiento para el sector comunitario, a través del Fondo de Fomento Concursable de Comunicación Audiovisual (FOMECA). Con el claro objetivo de fortalecer al sector sin fines de lucro, dicho Fondo apuntaba a fomentar proyectos especiales de comunicación comunitaria y de pueblos originarios.

En términos fácticos, el específico sector de medios sin fines de lucro, entre los que se enmarcan los comunitarios, tiene presencia en la mayoría de las regiones del país y resulta muy heterogéneo, dinámico e inestable en términos de sostenibilidad y sustentabilidad de las propuestas comunicacionales.

Entre los medios comunitarios, la radio es la que presenta mayor expansión en términos cuantitativos y quizás sea el medio más horizontal en su estructura de propiedad, debido a la cantidad de emisoras existentes y a la cantidad de propietarios (Marino, Mastrini, Becerra, Rubini y Espada, 2015). Sin embargo, el acceso a la información vinculada a la existencia, funcionamiento y gestión de emisoras radiofónicas comunitarias resulta deficitario; debido a la escasez de información oficial y a las transformaciones permanentes que presenta el sistema de medios.

En relación a lo anterior, los estudios académicos orientados a conocer y analizar el desarrollo y expansión de las radios comunitarias resultan siempre susceptibles de actualización y, más aún en los últimos años, por los cambios desarrollados en términos mediáticos a partir de la sanción de la Ley ${ }^{\circ} 26.522$ (2009) y los posteriores decretos modificatorios impuestos desde inicios de 2016.

Las primeras investigaciones que buscaron mapear el sector radiofónico comunitario / alternativo sostienen que en la década de 1980 existían en Argentina centenares de emisoras que podían caracterizarse dentro del sector, aunque también se destaca la insuficiencia y la poca fiabilidad de los datos de esa época (Lamas, 2011; Guimerá, 2013; Villamayor, 2012). Asimismo, Ernesto Lamas (2011) describe cómo en la década de 1990 se produjo la desaparición de muchas radios comunitarias y alternativas producto del decomiso y las amenazas de cierre sobre algunas de ellas por parte de la gestión gubernamental del presidente Carlos Menem.

Una de las investigaciones más representativas previa sanción de la ley 26.522, determina que, según datos oficiales, en 2008 existían en la Argentina al menos 175 emisoras comunitarias (Becerra, Marino y Mastrini, 2015). En una misma línea, Analía Elíades (2009) explica que dicha cifra representaba el $9 \%$ de un total de 1940 frecuencias radiofónicas registradas por el Estado, a las cuales habría que sumarles un número indeterminado de frecuencias fuera de registro.

Ahora bien, en el escenario post sanción de la ley 26.522, es posible destacar la investígación de Judith Gerbaldo (2010) que permite acceder a una aproximación de la situación de 
las radios comunitarias vinculadas al Foro Argentino de Radios Comunitarias (FARCO). El relevamiento realizado a nivel país permite mapear las necesidades, problemas y propuestas de radios y actores sociales participantes en dichos espacios; y a su vez se logra realizar una tipificación de las principales características que agrupan en objetivos y experiencias a la mayor parte de las radios comunitarias argentinas, destacándose la promoción de la organización ciudadana y el fortalecimiento de la democracia. Sin embargo, la principal limitación del trabajo resulta la circunscripción del estudio a las emisoras asociadas a FARCO.

Asimismo, desde el Programa de Investigación sobre Industrias Culturales y Espacio Público de la Universidad Nacional de Quilmes, se realizó un estudio que relevó cada una de las decisiones adoptadas por la Autoridad Federal de Servicios de Comunicación Audiovisual (AFSCA) en relación al sector de medios sin fines de lucro. El estudio también cruzó esta información con el relevamiento de emisoras sin fines de lucro realizado por AFSCA en 2015 y por el censo del sector audiovisual hecho por AFSCA en 2010. También se incorporaron los datos del financiamiento del sector sin fines de lucro a través de los programas FOMECA (Marino, et al., 2015). Si bien el mapeo realizado resulta fundamental a nivel país, es importante remarcar que el relevamiento de medios no se realizó por el espíritu comunitario, en el sentido del rol social de las emisoras, sino por denominación en la razón social que indica la falta de ánimo de lucro.

En este punto cabe acotar que Jujuy (escenario donde se centra esta investigación) es una provincia que se encuentra a más de $1300 \mathrm{~km}$ de la capital argentina, en el extremo noroeste del país y limita al norte con el Estado Plurinacional de Bolivia; al oeste, con la República de Chile y al este y sur, con la provincia de Salta (Argentina). Históricamente el desarrollo de los medios de comunicación en nuestro país siguió un patrón con centro en Capital Federal, punto neurálgico del desarrollo de la nación argentina. Asimismo corresponde señalar que la llegada y expansión de la radio en Jujuy tuvo como factores determinantes tanto la orografía de la región (zona montañosa, al pie de la Cordillera de los Andes) como las políticas de comunicación que los diversos gobiernos aplicaron en materia de regulación de la radiodifusión.

Ante el panorama inaugurado con la ley 26.520, Jujuy -como ocurrió en el resto del país- asistió a un notable interés por el impulso de experiencias de radios de gestión privada sin fines de lucro (tal como la misma ley las caracteriza).

Ahora bien, en virtud de la heterogeneidad y dinamismo del sector de radios comunitarias, en este trabajo se consideró necesario un abordaje de investigación situado y que atienda a las particularidades socio-culturales de una provincia-región de frontera y periférica en relación al centro político-económico rioplatense argentino.

Desde ese enfoque, los antecedentes de investigación en el contexto regional del Noroeste Argentino permiten citar el trabajo de Mary Esther Gardella (2011) que presenta una cartografía de las emisoras comunitarias de la provincia de Tucumán, previa a la sanción de la Ley ${ }^{\circ}$ 26.522 (2009), que integran la Red de Frecuencia Solidaria y que más que responder a un proyecto comunicativo participativo y a un proyecto político transformador que ponga en discusión los relatos hegemónicos, constituyen pequeñas Pymes centradas en avisos, informaciones y necesidades cotidianas y cercanas a su comunidad. Asimismo, Gardella aborda el panorama posterior a la sanción de la Ley $n^{\circ} 26522$ de SCA (2009) y apunta que para las radios comunitarias el tema es desde dónde se construye esa radio, desde qué proyecto se paran ante las transformaciones histórico-sociales que los ubican como sujetos protagónicos y no como meros espectadores.

En el orden de la provincia de Jujuy, las primeras investigaciones revisten un carácter de relevamiento exploratorio de la cuestión. Así, Federico Noro (2012) propone repasar el cambio del marco legal-normativo argentino en 2009 y describe la experiencia de la FM Pachacuti, gestionada por la comunidad Coya de Cochinoca, como un caso representativo que explicita el modo en que la estructura de medios se fue modificando a partir de que las comunidades pueden acceder a la posesión de una licencia.

En sintonía con las líneas de estudio detalladas precedentemente, en el presente trabajo se expondrán los resultados del proyecto de investigación titulado "El mapa que faltaba. Cartografía empírica y dimensión estructural de las radios comunitarias en la provincia de Jujuy". Este proyecto se propuso como objetivo general relevar el universo de emisoras comunitarias que operan en el territorio de la provincia Jujuy y analizar sus condiciones de funcionamiento, durante los años 2016 y 2017. Y de manera específica, se pretendió identificar la cantidad de emisoras comunitarias y su ubicación geográfica; describir las estructuras de propiedad y las 
estrategias de financiamiento; las estructuras de gestión, organización y actores participantes. Por otra parte, también se buscó caracterizar las propuestas comunicativas y el perfil de cada radio.

La herramienta metodológica fundamental para acceder a la información necesaria para este trabajo fue la combinación de entrevistas sistematizadas $\mathrm{y} / \mathrm{o}$ cualitativas a responsables, encargados/as, coordinadores/as y/o actores participantes del proceso de organización y producción de las emisoras comunitarias. La elección de este instrumento se justifica ante la necesidad de poder conocer no solo información precisa sobre cada emisora, sino también conocer a través de los sujetos entrevistados cómo se representan a sí mismos y cómo analizan el mundo social y los detalles de su entorno, reevaluando el espacio inconsciente de su vida cotidiana (Sierra, 1998). Se buscó acceder a información de las emisoras a partir del relato de experiencias, fundamentos e interpretaciones de los sujetos protagonistas de los discursos radiofónicos.

\section{MAPEO DE EMISORAS COMUNITARIAS EXISTENTES EN JUJUY (2016-2017)}

Al igual que cualquier otro medio de comunicación, la radio es una institución social sujeta a leyes particulares, regulaciones, normas y cambios históricos propiciados por transformaciones en sus aspectos sociológicos, jurídicos y en las formas expresivas de sus discursos (Mata, 1993).

En Argentina, desde 2009 y a partir de la sanción de la Ley $n^{\circ} 26.522$ (2009) de Servicios de Comunicación Audiovisual, se reconocen legalmente tres tipos de prestadores de servicios radiofónicos (también válido para los audiovisuales): de gestión estatal, de gestión privada con fines de lucro y de gestión privada sin fines de lucro. Estos parámetros jurídicos definen el rango de posibilidades generales para la conformación institucional de las emisoras radiofónicas y en concreto orientan los principios específicos que guían sus prácticas comunicativas. En este sentido, en su capítulo "definiciones" la Ley precisa una conceptualización respecto a las emisoras comunitarias, definiéndolas como "actores privados que tienen una finalidad social y se caracterizan por ser gestionadas por organizaciones sociales de diverso tipo sin fines de lucro. Su característica fundamental es la participación de la comunidad tanto en la propiedad del medio, como en la programación, administración, operación, financiamiento y evaluación. Se trata de medios independientes y no gubernamentales. En ningún caso se la entenderá como un servicio de cobertura geográfica restringida" (Ley $\mathrm{n}^{\circ}$ 26.522, 2009). Sin embargo, la definición de lo comunitario excede a una ley que, es dable reconocerlo, registra su existencia pero no alcanza a ensayar un concepto que retrate la variedad y heterogeneidad de experiencias radiofónicas que se autodefinen como comunitarias.

Esa limitación conceptual que se destaca pretende recuperar y actualizar una dificultad recurrente en términos teóricos: cómo definir a las radios comunitarias. Varios autores (Vinelli y Rodríguez Esperón, 2004; Kejval, 2009) advierten acerca de la ausencia de una definición consensuada y representativa del sector comunitario. La indefinición podría sintetizarse en el hecho de que la utilización flexible del concepto referencia a prácticas comunicacionales de diversos tipos, a veces contradictorias, que responden a distintos proyectos político-culturales que se encaran desde lo radiofónico.

Ahora bien, como señala certeramente Kejval (2009), la falta de definiciones consensuadas sobre lo comunitario redunda en serias dificultades al momento de establecer criterios de inclusión y exclusión para definir un conjunto de prácticas comunicacionales como categoría; y, como ocurre en este trabajo, poder constituir un corpus de análisis. Por lo tanto, la posición aquí no es proponer un concepto a priori en torno a la comunicación comunitaria de las emisoras radiofónicas sino revisar las conceptualizaciones actuales de los propios medios, de las personas que forman parte de la gestión de las emisoras y allí advertir los puntos de articulación o zonas compartidas entre dichas apreciaciones y la enunciada por la ley de Servicios de Comunicación Audiovisual. En síntesis, el autoreconocimiento del carácter comunitario resultó el criterio medular para definir la inclusión de las emisoras en el corpus analítico.

Desde ese marco, el objetivo inicial de la investigación fue identificar la cantidad y detallar la ubicación de las emisoras comunitarias efectivamente existentes en la provincia de Jujuy. Dicho relevamiento se planificó inicialmente para el año 2016, pero las dificultades de acceso a la información pertinente demandaron que el relevamiento se extendiese hasta inicios del segundo cuatrimestre de 2017.

El trabajo de actualización de información del mapa de radios comunitarias de la provincia de 
Jujuy implicó un relevamiento de escucha de todo el dial, de las distintas localidades de la provincia de Jujuy, cuyo resultado implicó la configuración exploratoria del sistema radiofónico provincial, cuyo objetivo no estuvo contemplado inicialmente en el proyecto.

Dicho mapeo concluyó en el reconocimiento de 193 emisoras de radio identificables en el dial de las distintas localidades de la provincia de Jujuy. De ese total, 188 emisoras operaban en Frecuencia Modulada (FM) y 5 en Amplitud Modulada (AM). Se pudo concluir también, que del total de emisoras el 43,5\% funcionaba en la capital provincial. Por otra parte, fue posible destacar que el mapa radiofónico de Jujuy presentaba un escenario de profunda concentración comercial.

Ahora bien, a los efectos de cumplir con el primer objetivo propuesto para esta investigación se realizó una primera identificación de emisoras comunitarias apelando a dos estrategias de reconocimiento:

1) La autodefinición del carácter comunitario a partir del propio discurso radiofónico enunciativo (Mata y Scarafía, 1993) y

2) la aplicación de la técnica metodológica "bola de nieve" (Taylor y Bogdan, 1987) de consulta a responsables de emisoras que permitió identificar a aquellas radios dependientes de organizaciones sociales y que se autodefinieron bajo un espíritu comunitario en función de su rol social.

El resultado del relevamiento permitió concretar la sistematización del total de emisoras comunitarias que efectivamente estuvieron operativas en el periodo comprendido entre junio de 2016 y agosto de 2017.

En la tabla que se presenta a continuación se presenta dicho relevamiento, detallándose la denominación de las 18 emisoras sin fines de lucro caracterizadas como "comunitarias", su frecuencia de transmisión, el departamento y la localidad de funcionamiento de los estudios de transmisión, el estado de operatividad al momento del corte de la investigación y observaciones adicionales vinculadas al año de inicio de funcionamiento y propiedad.

Tabla 1. Detalle de radios comunitarias existentes en Jujuy (2016-2017)

\begin{tabular}{|c|c|c|c|}
\hline $\begin{array}{l}\text { Dpto. Provincial/ } \\
\text { Localidad }\end{array}$ & $\begin{array}{c}\text { Denomi- } \\
\text { nación }\end{array}$ & $\begin{array}{c}\text { Situación } \\
\text { operativa } \\
\text { Agosto } \\
2017\end{array}$ & $\begin{array}{c}\text { Observa- } \\
\text { ciones }\end{array}$ \\
\hline Cochinoca/Abra & FM & Funciona- & Emisora con \\
\hline
\end{tabular}

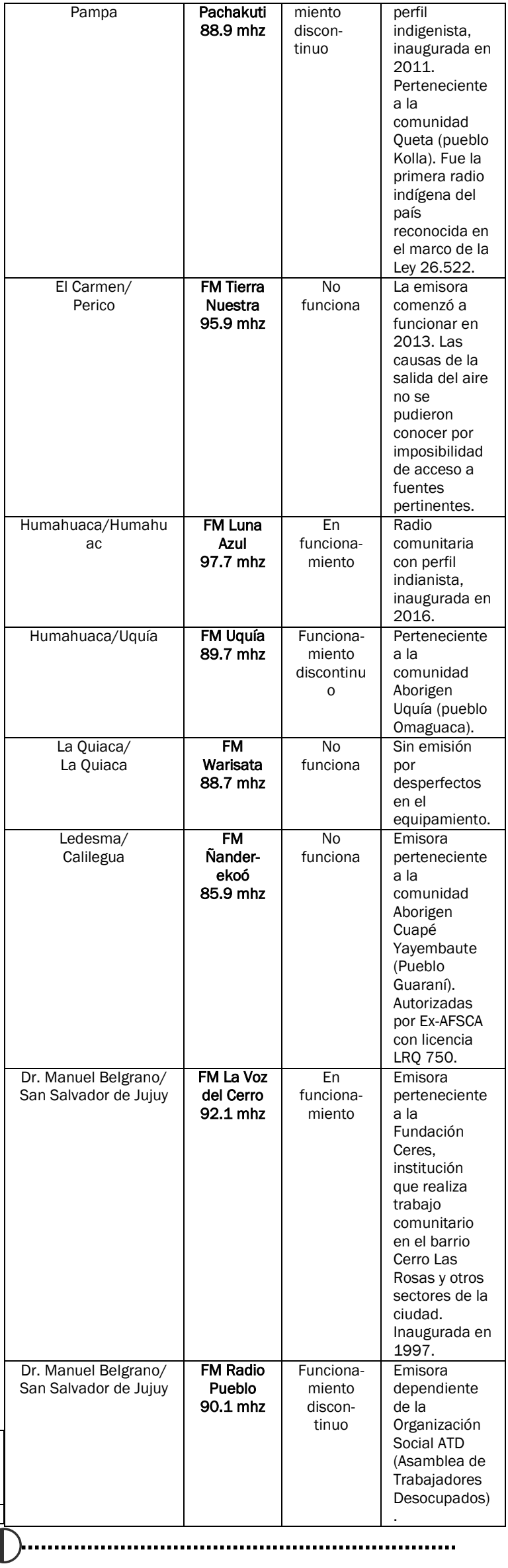


ARTÍCULOS

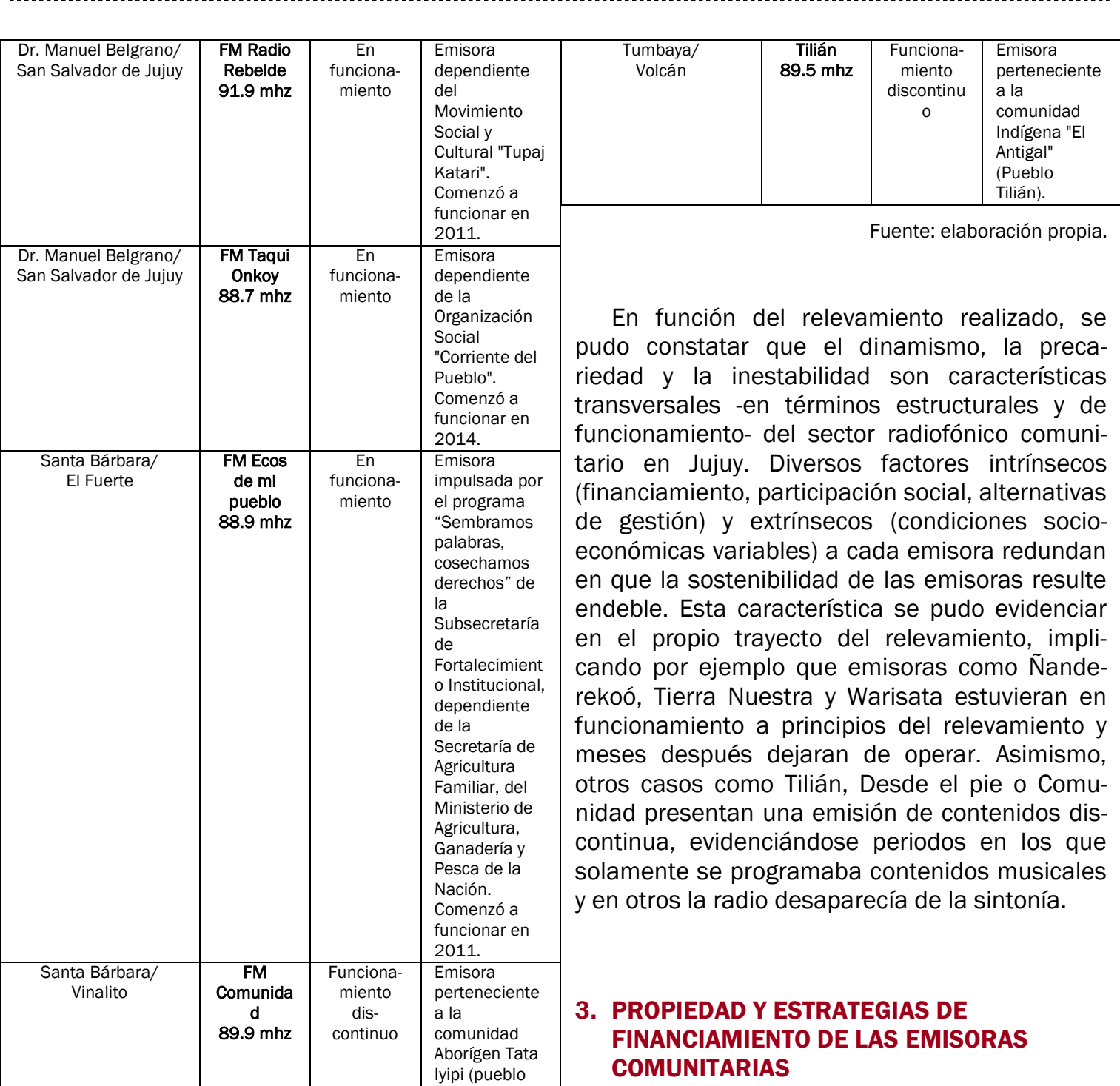

La institucionalidad de la radio en general supone un particular tipo de organización, que se torna en responsable de las instancias de administración, organización, producción, procesamiento y transmisión de los productos comunicacionales de la emisora radiofónica. Dicha organización estará sujeta a una estructura de propiedad, a estrategias organizativas internas, a un conjunto de recursos tecnológicos, a un sistema económico de financiación, a una determinada cantidad de personas vinculadas laboralmente, con formaciones profesionales particulares o la ausencia de ella, etc. La institucionalidad podrá estar vinculada también a un conjunto de objetivos y fines particulares, explícitos o implícitos, que se atribuirán a la institución radiofónica e incidirán necesariamente en las funciones que le serán atribuidas y las expectativas que surgirán de ellas (Avilés, 2014). 
Asimismo, la institucionalidad supone una posición ideológica que preside al emprendimiento, a cuyos preceptos el conjunto de actores radialistas adherirá total o parcialmente y se constituirán en factores condicionantes al momento de estructurar la dimensión discursiva. En este sentido, la institucionalidad radiofónica y todo lo que ella conlleva, influirá en la selectividad y orientación programática del discurso; el que no podrá conceptualizarse bajo ningún aspecto como etéreo, inmune o imparcial sino como una posición particular que, en coincidencia o disidencia con otras instituciones sociales, construirá parte de la realidad social a partir de su actividad discursiva (Avilés, 2014). En este sentido, la reflexión sobre la realidad y la experiencia comunitaria de las emisoras se convierte en una necesidad, para superar la concepción de la participación como una colección de opiniones. Para los casos en que la experiencia de comunicación va de la mano con una organización o movimiento social, se hace explícita además una intención de visibilización de clase, necesidades, intereses, actores, luchas que no son retratadas por los medios comerciales o estatales. La radio se presenta entonces como herramienta para darse a conocer y presentarse públicamente, configurando -desde el discurso radiofónico- una identidad positiva distinta de aquella que ofrecen los "medios masivos" locales (radios comerciales y/o estatales, canales de televisión, prensa escrita).

Desde dicha perspectiva, otro de los objetivos que se definieron para esta investigación fue caracterizar las estructuras de propiedad y las estrategias de financiamiento de las radios comunitarias relevadas en la provincia de Jujuy.

En función de lo expresado por los/as propios/as radialistas entrevistados/as, la idea de una propiedad compartida resulta un posible eje en torno al cual se construye la noción de lo comunitario vinculado al medio. Propiedad que no se circunscribe al equipo que trabaja y pone al aire la propuesta discursiva, sino que se hace extensiva al barrio o a la comunidad en el cual se encuentra emplazada la emisora.

Ahora bien, más allá de la conceptualización anterior, en términos de propiedad formal se pudo constatar que el 90\% (14 radios) de las emisoras comunitarias relevadas, y que se encontraban en funcionamiento al momento del cierre de la investigación, se organizaban y enmarcaban en torno a figuras jurídicas cuya principal característica era la de no perseguir fines de lucro: fundaciones, cooperativas o asociaciones civiles. Solo una sola emisora $(10 \%)$ funcionaba como sociedad de hecho y no registraba ninguna personería jurídica que encuadrara legalmente a la radio.

A su vez, las estructuras de propiedad y organización se pudieron clasificar en torno a tres grandes ejes:

1) Radios que nacieron y subsistían como emisoras comunitarias por la iniciativa de ciertos grupos sin una pertenencia institucional concreta: grupo de vecinos/as o grupo de amigos/as, con ideales particulares compartidos;

2) emisoras de comunidades aborígenes y que se pensaron como una herramienta comunicacional de dicha comunidad (FM Pachakuti, FM Luna Azul, FM Comunidad, FM Whipala, y

3) emisoras que nacieron y estructuraron como parte de organizaciones o movimientos sociales pre-existentes y el proyecto radiofónico se tradujo en un complemento o acción comunicacional de dicha organización; al margen de que luego la radio adquiriera una relativa autonomía. Esta última caracterización se aplica a los casos de Radio Pueblo, Radio Rebelde y FM Taqui Onkoy.

En relación a lo anterior y en virtud de reconocer que el sector de radios comunitarias resulta particularmente vulnerable a las condiciones sociales y económicas, en la investigación se pudo advertir un segmento de emisoras que logró una subsistencia estable durante muchos años: FM La Voz del Cerro (20 años de funcionamiento); FM Azul (17 años de funcionamiento); FM Pachakuti, Radio Rebelde, FM Ecos de mi Pueblo y FM Comunidad (todas con 6 años de funcionamiento). El resto de las emisoras relevadas representaban proyectos con un fuerte impulso inicial pero que ante el surgimiento de dificultades estructurales o coyunturales (propias o ajenas a la emisora) la cohesión grupal tendía a relajarse y, en consecuencia, el proyecto comunitario se debilitaba o desaparecía. Esta distinción propuesta, entre las diferencias en la subsistencia de las emisoras de radio comunitaria, resulta un aspecto necesario de ser estudiado con mayor profundidad considerado en futuras investigaciones que se ocupen de analizar las específicas condiciones de sostenibilidad de los proyectos radiofónicos comunitarios, atendiendo además del aspecto económico a dimensiones culturales, éticas, políticas y sociales.

Ahora bien, en términos de financiamiento y sustentabilidad económica, las radios comunitarias se inscriben, en el marco de la Ley $n^{\circ}$ 26.522 (2009) y en la definición de sus radia- 
listas, dentro de la categoría de emisoras sin fines de lucro. Esta categoría, supone y obliga a los/as radialistas comunitarios/as a producir contenidos sin la pretensión de obtener ningún tipo de utilidad económica a cambio de sus producciones comunicacionales. Sin embargo, el financiamiento y sustentabilidad de las emisoras dependen necesariamente de la obtención de recursos económicos para afrontar diversos costos de funcionamiento. Esta realidad obviamente es compartida, en niveles diferentes, con las emisoras con fines de lucro y estatales.

En función de las particularidades del sector radiofónico comunitario se puede aseverar que los costos de funcionamiento de las emisoras se concentran en dos categorías:

1) Costos fijos: representados principalmente por el pago del alquiler del espacio de funcionamiento, salvo en los casos en los que las emisoras funcionan en espacios cedidos o prestados sin la necesidad de contraprestación económica (espacios pertenecientes a municipios, centros vecinales $u$ otras instituciones estatales), $y$

2) costos variables: ligados al pago de servicios públicos o particulares necesarios para la producción, como electricidad, agua, teléfono, Internet.

Asimismo, resulta importante aclarar que en el total de las emisoras relevadas la variable honorarios o sueldos del personal de la emisora no representaba un costo considerado, ya que la sostenibilidad de la emisora -en términos de producción de contenidos y desarrollo de programas- dependía exclusivamente del trabajo y la participación voluntaria y ad honorem de los/as integrantes estables $\mathrm{y} / \mathrm{u}$ ocasionales vinculados/as a la emisora.

Asimismo, salvando particularidades inherentes a cada experiencia radiofónica y su condición institucional, en el trabajo de investigación se pudo reconocer al menos cuatro estrategias de financiamiento compartidas, en términos mayoritarios, por todas las emisoras comunitarias para lograr el funcionamiento cotidiano:

>> Aportes $y / 0$ acciones recaudatorias de los/as propios/as radialistas: El sostenimiento financiero de diversas emisoras comunitarias jujeñas dependía de manera parcial o total del aporte solidario de los/as propios/as radialistas. Dicho aporte se traducía en una cuota monetaria mensual o bien en aportes ocasionales y adicionales en función de necesidades específicas, como por ejemplo la reparación o reposición de algún elemento tecnológico de la emisora. Este recurso solía complementarse con acciones desvinculadas de lo radiofónico, organizadas para la recaudación de fondos: rifas, festivales, conciertos, venta de productos usados, venta de comida, concursos, entre otras.

>> Venta de espacios publicitarios: El financiamiento mediático mediante la venta de espacios publicitarios resulta la principal estrategia utilizada por los medios de comunicación con fines de lucro y una alternativa posible de utilización por parte de los medios estatales. Sin embargo, la utilización de esta estrategia de financiamiento por parte de las radios comunitarias jujeñas conlleva muchas veces un debate ideológico e identitario. Se pudo advertir que este debate, en niveles diferentes, atravesaba y tensionaba casi a la mitad de las emisoras comunitarias de Jujuy y el eje central de dicho debate estaba orientado a poder discernir si la venta de espacios publicitarios afectaba $\mathrm{y} / \mathrm{o}$ contradecía los principios asociados al carácter "comunitario" de las radios. En este sentido, para los/as radialistas comunitarios/as cónsultados/as, la venta publicitaria representaba una práctica íntimamente ligada con las radios comerciales (con fines de lucro) de las cuales intentan diferenciarse, por cuestiones políticas e ideológicas. Dicho afán de distanciamiento llevó a que algunas emisoras comunitarias hayan decidido prescindir por completo de la publicidad como estrategia de financiamiento.

En otras emisoras comunitarias la venta de espacios publicitarios resultaba una práctica aceptada pero siempre que se enmarcara y se respetaran ciertos criterios pre-acordados que, de alguna manera, servían como argumentos que morigeraban la tensión de tener que recurrir a una práctica tan vinculada, según los/as radialistas, a las radios comerciales. De esta manera, el criterio utilizado respecto a la publicidad pretendía marcar una diferencia entre las prácticas institucionales y las prácticas individuales de aquellas personas que utilizaban el espacio radiofónico para la realización de programas. En este sentido, puede interpretarse que la venta publicitaria se aceptaba bajo la condición de que la práctica no sea en beneficio de grandes comercios sino que permitiera beneficiar a pequeños negocios o a comerciantes ambulantes vinculados al barrio o a la comunidad donde tuviera injerencia la emisora. 0 bien que los comercios auspiciantes compartieran con la radio una misma condición de clase socio-económica, que represente un beneficio compartido y de carácter socio comunitario.

>> Financiamiento compartido con otra institución y actividades alternativas asociadas a 
ella: Finalmente, entre las radios comunitarias relevadas para este trabajo se pueden mencionar casos específicos de emisoras que surgieron y dependían de una organización social, con la cual compartían diversos recursos que colaboraban con el específico financiamiento de la emisora. Asimismo, la relación de muchos/as integrantes de las organizaciones con la radio posibilitaba el desarrollo de diversas actividades alternativas a la producción radiofónica que procuraban la generación de recursos económicos para colaborar con su mantenimiento: fiestas infantiles, alquiler de equipamientos de sonido, obtención de donaciones voluntarias, entre otras alternativas.

>> Financiamiento estatal: Las fuentes de financiamiento ligadas al Estado resultan otra manera a partir de las cuales las radios comunitarias pueden obtener recursos económicos. Sin embargo, esta alternativa no resulta una fuente de recursos que pueda considerarse estable y mucho menos confiable en términos de acceso. Igualmente, emisoras comunitarias como La Voz del Cerro, Pachakuti y Ecos de mi Pueblo comenzaron a funcionar a partir del acceso a equipamientos tecnológicos conseguidos por gestiones realizadas ante representantes del Gobierno Nacional. Sin embargo, el acceso al financiamiento a través de organismos del Estado implica, para las radios comunitarias, cumplir ciertos requerimientos como la vinculación a organizaciones facilitadoras como FARCO o bien estar reconocidas jurídicamente, lo que muchas veces no se corresponde con la realidad de las emisoras comunitarias de distintos lugares de la provincia. Un ejemplo de esto último resulta la posibilidad o no de poder participar en el Fondo de Fomento Concursable para Medios de Comunicación Audiovisual (FOMECA), un mecanismo de financiamiento previsto en el artículo 97 de la Ley $n^{\circ} 26.522$ (2009) de Servicios de Comunicación Audiovisual pensado para fortalecer a los actores de la comunicación sin fines de lucro. Sin embargo, como relataron varios actores de radios comunitarias jujeñas, existen limitaciones como la falta de licencia radiofónica, la necesidad de una cuenta bancaria activa, exigencias de facturación y otros requerímientos que limitan u obstaculizan las presentaciones de las emisoras a estos mecanismos concursables de fomento. Por otra parte, y en una línea similar a lo explicado en relación a la venta de espacios publicitarios, existen posicionamientos ideológicos de algunos/as radialistas comunitarios/as que interpretan que un elemento identitario central de este tipo de emisoras implica un distanciamiento absoluto de cualquier organismo y/o recurso proveniente del Estado.

\section{FORMAS DE ORGANIZACIÓN, GESTIÓN Y ACTORES PRINCIPALES}

En este apartado se presentan los resultados y conclusiones arribados en virtud de la pretensión de caracterizar las estructuras de gestión, organización y actores participantes en las emisoras comunitarias relevadas en la provincia de Jujuy.

Como se detalló anteriormente, en términos de organización y gestión, el $90 \%$ de las emisoras comunitarias relevadas y que se encontraban en funcionamiento al momento del cierre de la investigación se organizaban en torno a figuras jurídicas cuya principal característica era la de no perseguir fines de lucro: fundaciones, cooperativas o asociaciones civiles.

Dicho encuadre legal, en cierto modo, se convenía con la organización para el funcionamiento de las radios, traducido en la colaboración de sus integrantes para el logro de los objetivos propuestos, el que no necesariamente es compartido y conocido en profundidad por el total de los miembros participantes, pero que en gran medida atravesaba el perfil de cada emisora. En este sentido, el espíritu de colaboración mutua y la creación de un patrimonio común irrepartible resultaba una característica transversal a todas las emisoras comunitarias.

En términos estrictamente organizativos, se pudo concluir que las radios comunitarias jujeñas funcionaban -en el período analizado- con una estructura en la que se podían diferenciar tres formas de integración y participación de sus miembros:

>> Coordinación General: con esta categoría se puede unificar a las formas organizativas que nuclean al grupo de integrantes de las emisoras encargado de administrar, gestionar y tomar decisiones operativas cotidianas para garantizar el funcionamiento de la radio. En las diferentes emisoras este formato organizativo podía adquirir nominaciones particulares, "comisión Interna" (FM Caprichosa) "equipo responsable" (Radio Rebelde), pero en todos los casos coincidían en los objetivos. Por otra parte, otro punto de coincidencia entre las emisoras comunitarias, se manifestaba en las condiciones internas establecidas para poder integrar esa Coordinación General, la que se traducían en el reconocimiento de aquellos miembros que evidenciaran 
un trabajo "activo" y una participación "estable" y "constante" en el funcionamiento de las radios. En todos los casos, se advirtió la intención de mantener un trabajo horizontal, en el que la toma de decisiones cotidianas resultara de reuniones semanales o eventuales según demandara la situación. En dichas reuniones se buscaba la participación de dicho grupo reducido de integrantes de la radio.

A su vez, en algunos casos particulares ( $\mathrm{La}$ Voz del Cerro, Radio Pueblo, FM Taky Onqoy), dicha coordinación general se subdividía en áreas específicas que asumían responsabilidades concretas, como por ejemplo gestión de contenidos, atención de equipamientos técnicos, vinculaciones externas, programación y acompañamiento, finanzas, producción, programación y educación, armado de proyectos, convenios institucionales, pago de servicios.

>> Asambleas / reuniones colectivas: Por otra parte, la toma de decisiones que implicaban asuntos que excedían cuestiones operativas cotidianas, se desarrollaban bajo el formato de asambleas o reuniones colectivas en las que se abría la participación al total de las personas que tenían vinculación con las radios comunitarias. En términos generales, según lo expresado por los/as entrevistados/as, la convocatoria a dichas reuniones respondía a alguna temática en particular y las decisiones de las acciones a seguir se las debatía hasta llegar a acuerdos colectivos o bien se sometían a votación cuando los consensos no se alcanzaban. Estos espacios servían además para poner en común información sobre proyectos iniciados, aspectos operativos cotidianos y realizar ciertos balances de gestión.

>> Participación en la producción / realización de programas: a su vez, se pudo diferenciar al resto de les actores participantes de las emisoras, quienes no tenían un vínculo necesariamente estable con la misma, ni integraban el equipo de coordinación, pero sí participaban de manera, más o menos constante, en la realización de los programas radiofónicos que integraban las grillas de programación.

Por otra parte, se pudo concluir que la cantidad de personas que participaban activamente en el funcionamiento de las radios comunitarias jujeñas estudiadas era de un promedio de entre 7 y 13 personas. Igualmente, un dato que resultó significativo, fue que los/as entrevistados/as no pudieron precisar en ningún caso la cantidad de participantes que se involucraban en el cotidiano funcionamiento de la radio. Esta imprecisión se debió a que muchos/as de dichos actores solían actuar esporádicamente como colaboradores en la producción de los programas de radio y su vínculo con la emisora no estaba consolidado.

Como se advirtió precedentemente, la participación de las personas vinculadas con las radios para la producción y realización de programas resultaba en todos los casos un trabajo voluntario y ad honorem.

En ese sentido, el factor de "voluntariado" atentaba muchas veces contra la continuidad y estabilidad de los programas, ya que la participación y producción en la radio no se interpretaba unificadamente como una responsabilidad estable, o bien la satisfacción de otras necesidades personales llevaba a que los/as integrantes de las emisoras interpretaran o asumieran la realización radiofónica como una actividad secundaria y prescindible.

\section{PROPUESTAS COMUNICATIVAS Y PERFIL DE LAS EMISORAS}

Por último, entre los objetivos del proyecto de investigación se pretendió realizar el análisis de la dimensión estructural del discurso de cada radio comunitaria, con el fin de poder caracterizar su organización de programación, no sólo en términos temporales sino también en la jerarquización de contenidos, programas o la inclusión de determinados elementos discursivos distintivos y específicos (Mata y Scarafía, 1993).

A partir del acceso a información de las estructuras de programación y en base a las entrevistas realizadas a radialistas del sector radiofónico comunitario de Jujuy resulta posible presentar algunos resultados exploratorios que permiten cumplimentar parcialmente los objetivos previstos.

En primer lugar, se pudo establecer una primera clasificación de la situación de las emisoras comunitarias jujeñas en virtud de su situación en el desarrollo de programación, no sólo en producción de contenidos y puesta al aire de programas. En este sentido, al momento del cierre la investigación, la situación operativa de las emisoras se pudo diferenciar en tres categorías:

>> Con funcionamiento pleno: en esta categoría se enmarcaban aquellas emisoras comunitarias que contaban con una programación com- 
pleta y transmisión de contenidos de hasta 18 horas diarias. Las emisoras que entraban en esta clasificación fueron siete: FM Luna Azul, FM La Voz del Cerro, FM Radio Rebelde, FM Taqui Onkoy, FM Ecos de mi pueblo, Radio La Caprichosa, Azul FM y FM Wiphala

>> Con funcionamiento discontinuo: en esta categoría se enmarcaban las emisoras cuya programación resultaba inestable e intermitente. Esto implicaba que los programas que se ponían al aire no presentaban una continuidad, habitualmente cambiaban de horarios y/o días, existían franjas horarias o días completos en las que la emisora dejaba de transmitir o simplemente se programaban contenidos musicales y también se realizaban coberturas informativas excepcionales según acontecimientos de interés para los/as productores/as de la radio. Las emisoras que se clasificaban en esta categoría fueron seis: FM Pachakuti, FM Uquía, FM Radio Pueblo, FM Comunidad, Radio Desde el pie y FM Tilián.

>> Dejaron de transmitir: En esta categoría se incluyó a las emisoras comunitarias que en algún periodo del proceso de relevamiento estuvieron funcionando con programación (ya sea musical o con programas de contenidos) pero al momento del cierre de la investigación se pudo constatar su absoluta ausencia de transmisión. Las emisoras en esta categoría fueron tres: FM Tierra Nuestra, FM Warisata, FM Ñanderekoó.

Por otra parte, considerando solamente aquellas emisoras que contaban con programación estable, se pudo caracterizar dos perfiles de propuestas comunicativas que, en términos generales, compartirían las emisoras comunitarias:

>> En función de los intereses de los/as radialistas participantes: Bajo esta categoría se pueden agrupar a las emisoras que organizaban sus propuestas de contenidos en función de las iniciativas individuales o grupales de personas de la comunidad, el barrio o el pueblo. Un ejemplo claro lo constituía FM La Caprichosa, ya que en las consultas realizadas se manifestó que "el perfil de la radio se arma con lo que la comunidad le interesa compartir: programas de selección musical del dueño de una ferretería, propuestas musicales de bandas independientes, propuestas musicales alternativas a la música comercial. Otros programas que tienen continuidad buscan comunicar sobre la realidad de lo que es Tilcara, cosmopolita, diversa, que tiene distintas identidades". Asimismo, los casos de FM Azul, Ecos de mi Pueblo y La Voz del Cerro, organizaban la propuesta de contenidos con una lógica similar, poniendo a disposición la emisora para que los/as participantes pudieran compartir información que favoreciera, contribuyera y empoderara a la comunidad pero también que respondiera a los gustos y consumos culturales asociados al entretenimiento. En este sentido, las propuestas comunicativas integraban tanto información periodística local y de producción propia, con información nacional e internacional, como también programas juveniles, deportivos, de música romántica, tropical, folclore, entre otras propuestas. Cabe destacar también la presencia de propuestas de contenidos educativos y culturales.

>> Enmarcadas en un perfil ideológico predefinido: Si bien las emisoras que se agrupan en esta categoría pueden presentar coincidencias con las anteriores en materia de diversidad de propuestas de contenidos, el elemento diferencial responde a un mayor énfasis marcado por la coordinación general de las radios en que los programas $\mathrm{y} / \mathrm{o}$ contenidos se adecuaran a un perfil preestablecido. En general, dicho perfil respondía a un posicionamiento político-ideológico consecuente con la organización social o la comunidad a la que se vinculaba la emisora. Por ejemplo, las emisoras comunitarias Radio Pueblo, Radio Rebelde y Taki Onkoy buscaban construir sus programaciones y propuestas comunicativas en función de dos estrategias: por un lado facilitar la comunicación de las actividades propias del movimiento y organización social para mantener un vínculo con los barrios y servir de herramienta política, y, por otro lado, construir una agenda informativa de producción local que resultara alternativa a la de los medios con fines de lucro locales, bajo el argumento de que estos medios invisibilizan acontecimientos de interés social. Asimismo, en el caso de Radio Luna Azul, los/as integrantes consultados/as para este trabajo manifestaron que resultaba requisito esencial para formar parte de los programas de la radio presentar un proyecto que se integrara y respetara el perfil cultural e identitario de la radio: la ideología indianista.

\section{CONCLUSIONES}

¿Y el mapa que faltaba? De un mapa se espera certezas, que indique por dónde ir y permita localizar con acierto determinados hitos (reales, simbólicos, comunicacionales, en este caso). 
Pero una de las conclusiones a las que esta investigación ha permitido arribar es que el mapa -tal como se pudo imaginar antes de emprender el relevamiento- brindará caminos varios, cambiantes, pero no definiciones absolutas.

La comunicación en Argentina, en general, y la radio y la comunicación comunitaria en particular, asisten a una serie de embates que arrastran desde antes de la sanción de la Ley de Servicios de Comunicación Audiovisual. Dichos embates pretendieron ser atendidos a través de la Ley $n^{\circ} 26.522$ (2009) en algunos aspectos desde 2009, pero la insuficiente implementación de la Ley dejó al ecosistema de medios sin fines de lucro expuesto ante la inminencia de un nuevo giro en materia de comunicación que representaría una profundización de la inestabilidad del sector.

En ese orden, como se mencionó anteriormente, la disolución de la Autoridad Federal de Servicios de Comunicación Audiovisual (AFSCA), en enero de 2016, y otras medidas implementadas por decreto por el recién asumido presidente Mauricio Macri, anticiparon a las radios comunitarias una serie de modificaciones que las afectarían directamente y pondrían en jaque su continuidad.

Del relevamiento realizado, se puede tener pistas de cómo sobrevivían y se organizaban en el período analizado, pero ante la inestabilidad y precariedad del sector se desprende que el mapa puede haber perdido actualidad para dar cuenta de las emisoras que continúan al aire.

Ahora bien, si la sustentabilidad económica de un proyecto radiofónico de esta naturaleza resulta difícil de garantizar en condiciones más o menos estables, en el marco de una crisis económica como la que se vive en Argentina, la supervivencia -si se sostiene- busca asentarse en la sostenibilidad del proyecto de comunicación que orienta las prácticas.

De regreso en los temas abordados por este trabajo y los resultados obtenidos, vale decir que aún quedan por responder preguntas que emergen y reclaman tratamiento académico, como, por ejemplo: ¿cómo se construye y sostiene la horizontalidad pretendida en las radios que forman parte de organizaciones sociales? ¿Qué lugar ocupan o se les concede a las mujeres en esos medios y por qué? ¿Logran las radios comunitarias jujeñas escapar a la lógica del patriarcado que permea casi todas las prácticas culturales en la provincia? ¿Cómo se relacionan con la comunidad de oyentes las radios comunitarias? ¿A quién/es y cómo le/s hablan?

Un mapa de preguntas, de nuevos cuestionamientos, un mapa en el que los mojones o señales se mueven todo el tiempo y requieren la constante atención de quienes estudiamos, enseñamos, investigamos y compartimos experiencias de extensión universitarias. Así es el mapa que acá se propone.

\section{NOTAS}

${ }^{1}$ Se utiliza la expresión en términos potenciales debido a que la reserva de dicho $33 \%$ de las licencias, en todas las bandas, frecuencias y zonas geográficas, nunca logró ser aplicado.

2 La determinación de estas tres categorías se logró, en términos metodológicos, a partir de dos instancias combinadas de consulta informativa: el contacto con personas vinculadas a las emisoras y el chequeo de la señal de transmisión. Fundamentalmente para aquellas tres emisoras incluidas en la tercera categoría, la confirmación por parte de personas vinculadas a las radios resultó fundamental.

\section{REFERENCIAS BIBLIOGRÁFICAS}

- Avilés Rodilla, C. (2014). Radio estataly ciudadanía. Tensiones, articulaciones y contradicciones en torno al rol de la radio de propiedad estatal en San Salvador de Jujuy. (Tesis Doctoral). La Plata: Universidad Nacional de la Plata.

- Eliades, A. (2009). El derecho a comunicar y la actividad radiodifusora. La Plata: Universidad Nacional de La Plata.

- Gardella, M. E. (18-29 de mayo de 2011). Lo que se escucha en las radios de Tucumán. Cambios operados a partir de la ley 26.522. S.p.. Memorias de las X Jornadas regionales de Investigación en Humanidades y Ciencias sociales. Facultad de Humanidades y Ciencias Sociales, Universidad Nacional de Jujuy, Argentina.

- Gerbaldo, J. (2010). Hacia una cartografía de las radios comunitarias argentinas. Revista Diálogos de la Comunicación, 8, 1-8. Recuperado de: http://dialogosfelafacs.net/edicion-82/

- Guimerá, J. A. (2013) Políticas de comunicación, pluralismo y televisión sin afán de lucro: el caso de Argentina. Ámbitosdecomunicación.com, 23. Recuperado de: http://institucional.us.es/ambitos/?p=623

- Iglesias, M. (2015). A contramano: modelos de gestión, modos organizativos y estrategias 
económicas de las emisoras comunitarias argentinas en búsqueda de la sustentabilidad (2005-2015) (Tesis de Maestría). Universidad Nacional de Quilmes, Bernal, Bs.As, Argentina. Recuperado de: https://cpr.org.ar/wpcontent/uploads/media/uploads/documents/inve stigacionpidc/martin-iglesias-a-contramano-micc.pdf

- Kejval, L. (2009). Truchas. Los proyectos políticoculturales de las radios comunitarias, alternativas y populares. Buenos Aires: Prometeo.

- Lamas, E. (2011). Medios audiovisuales comunitarios: legitimidad y legalidad. Un reconocimiento merecido. En Baranchuk, M. y Rodríguez Usé, J. (coord.). Ley 26.522. Hacia un nuevo paradigma en comunicación audiovisual (p.p. 143-159). Lomas de Zamora, Bs.As, Argentina: AFSCA - UNLZ

- Ley № 26.522 Servicios de Comunicación Audiovisual. Boletín Oficial de la República Argentina. Buenos Aires, Argentina. 10 de octubre de 2009. Recuperado de: http://servicios.infoleg.gob.ar/infolegInternet/ane xos/155000-159999/158649/norma.htm.

- Marino, S., Mastrini, G., Becerra, M., Rubini, C. y Espada, A. (2015). Diagnóstico sobre el acceso del sector sin fines de lucro a medios audiovisuales en la Argentina 2014. Licencias, autorizaciones, permisos y fondos concursables. Universidad Nacional de Quilmes: Bernal, Bs.As., Argentina. Recuperado de: https://martinbecerra.files.wordpress.com/2015/ 03/diagnostico-medios-sin-lucro-unq-icepmar2015.pdf.

- Mata, M. C. (1993). La radio una relación comunicativa. Revista Diálogos de la Comunicación, 35.10-13. Recuperado de:

http://dialogosfelafacs.net/edicion-35/.

- Mata, M. C. y Scarafía, S. (1993). Lo que dicen las radios. Una propuesta para analizar el discurso radiofónico. Quito: Aler.

- Noro, A. F. (2012). Radio "Pachacuti”: primera radio FM indígena de la Argentina. Revista Question, 1 (35), 401-410. Recuperado de: https://perio.unlp.edu.ar/ojs/index.php/question/ issue/view/72

- Open Society Foundations (2012). Mapping digital media: Argentina. (Country Report, february, 29), London: OSF. Recuperado de: http://www.opensocietyfoundations.org/sites/def ault/files/mapping-digital-media-argentina20121107.pdf.

- Sierra, F. (1998). Función y sentido de la entrevista cualitativa en investigación social. En: Galindo, J. (coord.), Técnicas de investigación en sociedad, cultura y comunicación. (pp. 277-346). México: Editorial Pearson.
- Taylor, S. y Bogdan, R. (1987). Introducción a los métodos cualitativos de investigación. La búsqueda de significado. Barcelona: Paidós.

- Villamayor, C. (2012). Planificar y gestionar desde una perspectiva comunicacional. Documentos del Curso Planificación y Gestión de procesos Comunicacionales II. Maestría PLANGESCO. Facultad de Periodismo y Comunicación Social. Universidad Nacional de La Plata, La Plata, Argentina.

- Vinelli, N. y Rodríguez Esperón, C. (2004). Contrainformación. Medios alternativos para la acción política. Buenos Aires: Ediciones Continente. 